\title{
Association of the Modified ALBI Grade With Endoscopic Findings of Gastroesophageal Varices
}

\author{
YUHO MIYAMOTO $^{1,2}$, HIRAYUKI ENOMOTO ${ }^{1}$, HIROKI NISHIKAWA ${ }^{1}$, \\ TAKASHI NISHIMURA ${ }^{1}$, YOSHINORI IWATA ${ }^{1}$, SHUHEI NISHIGUCHI ${ }^{3}$ and HIROKO IIJIMA ${ }^{1}$ \\ ${ }^{I}$ Division of Gastroenterology and Hepatology, Department of Internal Medicine, \\ Hyogo College of Medicine, Nishinomiya, Japan; \\ ${ }^{2}$ Department of Gastroenterology, Takarazuka City Hospital, Hyogo, Japan; \\ ${ }^{3}$ Department of Gastroenterology, Kano General Hospital, Osaka, Japan
}

\begin{abstract}
Background/Aim: This study aimed to assess the association of the modified albumin-bilirubin (mALBI) grade with the endoscopic findings of gastroesophageal varices (GEVs). Patients and Methods: A total of 141 patients with histologically proven cirrhosis who underwent a liver biopsy and esophagogastroduodenoscopy were enrolled. The relationships between the $m A L B I$ grade and endoscopic findings were evaluated. Results: The incidence of GEVs and high-risk GEVs differed among mALBI grades. Patients with $m A L B I$ grades of $2 b-3$ had higher rates of GEVs and highrisk GEVs in comparison to those with $M A L B I$ grades of 1 $2 a(p<0.0001)$. In addition, patients with mALBI grade $2 b$ or grade 3, but not those with MALBI grade $2 a$, had significantly higher rates of complicated GEVs and high-risk GEVs in comparison to those with mALBI grade 1. Conclusion: The mALBI grade may be useful in predicting the presence of GEVs and for stratifying their bleeding risk.

Gastroesophageal varices (GEVs) are an important complication in patients with liver cirrhosis (1-3). Esophagogastroduodenoscopy (EGD) is the most reliable method for evaluating GEVs; however, from the viewpoint of the burden on patients, a non-invasive method for predicting the presence of varices, particularly those with a risk of hemorrhage, is desired $(4,5)$. Currently, a combination of liver stiffness data with transient
\end{abstract}

This article is freely accessible online.

Correspondence to: Hirayuki Enomoto, Division of Gastroenterology and Hepatology, Department of Internal Medicine, Hyogo College of Medicine, Mukogawa-cho 1-1, Nishinomiya, Hyogo 663-8501, Japan. Tel: +81 798456472, Fax: +81 798456474, e-mail: enomoto@hyo-med.ac.jp

Key Words: Liver cirrhosis, gastroesophageal varices, ALBI score. elastography (Fibroscan ${ }^{\circledR}$ ) and the platelet count is widely used as the established criteria (Baveno VI criteria) to determine the indication for EGD (6). However, the institutions that are able to perform transient elastography are limited; thus, the evaluation of varices based on blood test parameters is still clinically relevant. In particular, an accurate method of evaluation based on daily clinical data would be ideal.

The albumin-bilirubin (ALBI) grade is a newly reported classification that can be used to classify the liver reserve function into three grades (ALBI grades 1-3: i.e. from better to poorer function) using two commonly measured variables: The serum values of albumin and total bilirubin (7). However, ALBI grade 2 includes a wide-range of patients in whom the liver function is relatively heterogeneous, and a scoring system that combines the ALBI grade and platelet count (ALBI-PLT score) was reported to be useful for predicting the presence of high-risk GEVs in patients with hepatocellular carcinoma and compensated cirrhosis (8).

In order to alleviate the heterogeneity of patients with ALBI grade 2, a modified classification that divides ALBI grade 2 into two categories ( $a$ and $b$ ) was recently proposed as the 'modified ALBI grade' (mALBI grade) (9). However, the relationship between mALBI grade and the endoscopic findings of GEVs has not been clarified. In the present study, we assessed the mALBI grade, and evaluated their association with the endoscopic findings of GEVs.

\section{Patients and Methods}

We retrospectively studied a total of 141 patients who underwent a percutaneous liver biopsy at our Institution from October 2008 to December 2015 and who were pathologically confirmed to have liver cirrhosis. The etiologies of liver cirrhosis were determined and classified as described previously (10). Liver biopsy studies were conducted using standard techniques $(11,12)$. All blood data were obtained on the same day as the liver biopsies. The albumin level, total bilirubin level, and platelet count were measured in routine 
blood tests. The ALBI score (7), ALBI grade (7), ALBI-PLT score (8) and mALBI grade (9) were determined as follows:

ALBI score: [ $\log _{10}$ bilirubin $\left.(\mu \mathrm{mol} / 1) \times 0.66\right]-[$ albumin $(\mathrm{g} / \mathrm{l}) \times 0.085]$

ALBI grade by ALBI score: Grade $1: \leq-2.60 ; 2:>-2.60$ to $-1.39 ; 3:>-1.39$

ALBI-PLT score: Sum of points for ALBI grade (1-3 points) and platelet count ( 1 point: $>150 \times 10^{3} / \mathrm{mm}^{3} ; 2$ points: $\left.\leq 150 \times 10^{3} / \mathrm{mm}^{3}\right)$. ALBI-PLT score ranged from 2 to 5 .

mALBI grade: Grade 1: $\leq-2.60 ; 2 \mathrm{a}:>-2.60$ to $<-2.27 ; 2 \mathrm{~b}$ : $\geq-2.27$ to $-1.39 ; 3$ : $>-1.39$

GEVs were evaluated by EGD according to the current Japanese guidelines for the management of liver cirrhosis, as previously described $(3,13)$. Endoscopically detected GEVs were classified into three grades (F1, F2, or F3), and the presence of red signs on the varices was also investigated. Patients with $\mathrm{F} 2$ varices with red signs, and those with $\mathrm{F} 3$ varices were considered to have high-risk varices (13). Evaluation by EGD was conducted within 6 months of the liver biopsy. Patients who had previously received endoscopic treatment for varices were considered to have varices with a highrisk of bleeding.

Patients who had undergone splenectomy or partial splenic arterial embolization were excluded from the study, as such methods influenced the ALBI-PLT score. The study conformed to the ethical guidelines of the Helsinki declaration. The current study was approved by the Ethics Committee of the Institutional Review Board (No. 3431).

Statistical analysis. Frequencies were compared among the groups by the chi-squared test. Odds ratios between groups were determined with a logistic regression model (14). $p$-Values of less than 0.05 were considered to indicate statistical significance.

\section{Results}

Basic characteristics of patients with cirrhosis. A total of 141 patients with cirrhosis who were diagnosed by liver biopsy were enrolled; their basic characteristics are shown in Table I. The median age of the patients was 63 years. The study population included 73 men and 68 women. The distribution of the ALBI-PLT scores was as follows: score 2 in 10, 3 in 31, 4 in 95, and 5 in five. The distribution of the ALBI grades was as follows: Grade 1 in 25, grade 2 in 109, and grade 3 in seven. The 109 patients classified as grade 2 were further subdivided by the mALBI grade system into grade $2 \mathrm{a}$ in 40 and grade $2 \mathrm{~b}$ in 69 (Table I).

Association of the ALBI-PLT score with the endoscopic findings of GEVs. The incidence of GEVs increased as the ALBI-PLT score increased (overall: $p<0.01$ ) (Figure 1A). The incidence of high-risk varices also increased as the score increased, and among the patients with ALBI-PLT score 2, only one patient had high-risk varices; however, the differences among the groups (score 2-5) did not reach statistical significance (overall: $p=0.222$ ) (Figure 1B).

Association of the ALBI and $m A L B I$ grades with the endoscopic findings of GEVs. In the evaluation of ALBI
Table I. Characteristics of the enrolled patients with liver cirrhosis $(N=141)$.

\begin{tabular}{|c|c|c|}
\hline Characteristic & & Value \\
\hline Age, years & Median (IQR) & $63(54-70)$ \\
\hline \multirow[t]{2}{*}{ Gender, $\mathrm{n}$} & Male & 73 \\
\hline & Female & 68 \\
\hline \multirow[t]{9}{*}{ Etiology, $\mathrm{n}$} & $\mathrm{HBV}$ & 9 \\
\hline & $\mathrm{HCV}$ & 96 \\
\hline & $\mathrm{HBV}+\mathrm{HCV}$ & 1 \\
\hline & ALD & 13 \\
\hline & NASH & 6 \\
\hline & $\mathrm{AIH}$ & 7 \\
\hline & $\mathrm{PBC}$ & 1 \\
\hline & $\mathrm{AIH}+\mathrm{PBC}$ & 4 \\
\hline & Other & 4 \\
\hline \multirow{5}{*}{ Child-Pugh Score, $\mathrm{n}$} & 5 & 58 \\
\hline & 6 & 29 \\
\hline & 7 & 40 \\
\hline & 8 & 12 \\
\hline & 9 & 2 \\
\hline \multirow{2}{*}{$\begin{array}{l}\text { Past history of } \\
\text { hepatocellular carcinoma, } \mathrm{n}\end{array}$} & $(+)$ & 33 \\
\hline & $(-)$ & 108 \\
\hline $\operatorname{AST}(\mathrm{IU} / \mathrm{l})$ & Median (IQR) & $48(36-73.5)$ \\
\hline ALT (IU/l) & Median (IQR) & $41(26-64.5)$ \\
\hline$\gamma$-GTP (IU/l) & Median (IQR) & $47(30-89.5)$ \\
\hline $\operatorname{ALP}(\mathrm{IU} / 1)$ & Median (IQR) & $290(219.5-387.5)$ \\
\hline Albumin $(\mathrm{g} / \mathrm{dl})$ & Median (IQR) & $3.6(3.1-3.85)$ \\
\hline Total bilirubin (mg/dl) & Median (IQR) & $1.0(0.7-1.4)$ \\
\hline Platelet count $\left(\times 10^{3} / \mu \mathrm{l}\right)$ & Median (IQR) & $100(76.5-139)$ \\
\hline Prothrombin time $(\%)$ & Median (IQR) & $76.4(66.4-83.85)$ \\
\hline ALBI score & Median (IQR) & $-2.218(-2.518-1.811$ \\
\hline \multirow[t]{3}{*}{ ALBI grade, $n$} & 1 & 25 \\
\hline & 2 & 109 \\
\hline & 3 & 7 \\
\hline \multirow[t]{4}{*}{ mALBI grade, $n$} & 1 & 25 \\
\hline & $2 \mathrm{a}$ & 40 \\
\hline & $2 b$ & 69 \\
\hline & 3 & 7 \\
\hline \multirow[t]{4}{*}{ ALBI-PLT score, $\mathrm{n}$} & 2 & 10 \\
\hline & 3 & 31 \\
\hline & 4 & 95 \\
\hline & 5 & 5 \\
\hline
\end{tabular}

AIH: Autoimmune hepatitis; ALBI: albumin-bilirubin; ALBI-PLT: ALBI-platelet; ALD: alcoholic-related liver disease; ALP: alkaline phosphatase; ALT: alanine aminotransferase; AST: aspartate aminotransferase; $\gamma$-GTP: $\gamma$-glutamyl transpeptidase; HBV: hepatitis B virus; $\mathrm{HCV}$ : hepatitis $\mathrm{C}$ virus; IQR: interquartile range mALBI: modified ALBI; NASH: nonalcoholic steatohepatitis; PBC: primary biliary cholangitis.

grade alone, the incidence of GEVs statistically significantly differed among the groups, increasing with increasing grade (overall: $p<0.05$ ) (Figure 2A). The incidence of high-risk varices also statistically significantly increased as the grade increased (overall: $p<0.05$ ) (Figure 2B). When the mALBI grade was used, statistically significant differences were observed among the groups in the incidence of GEVs 

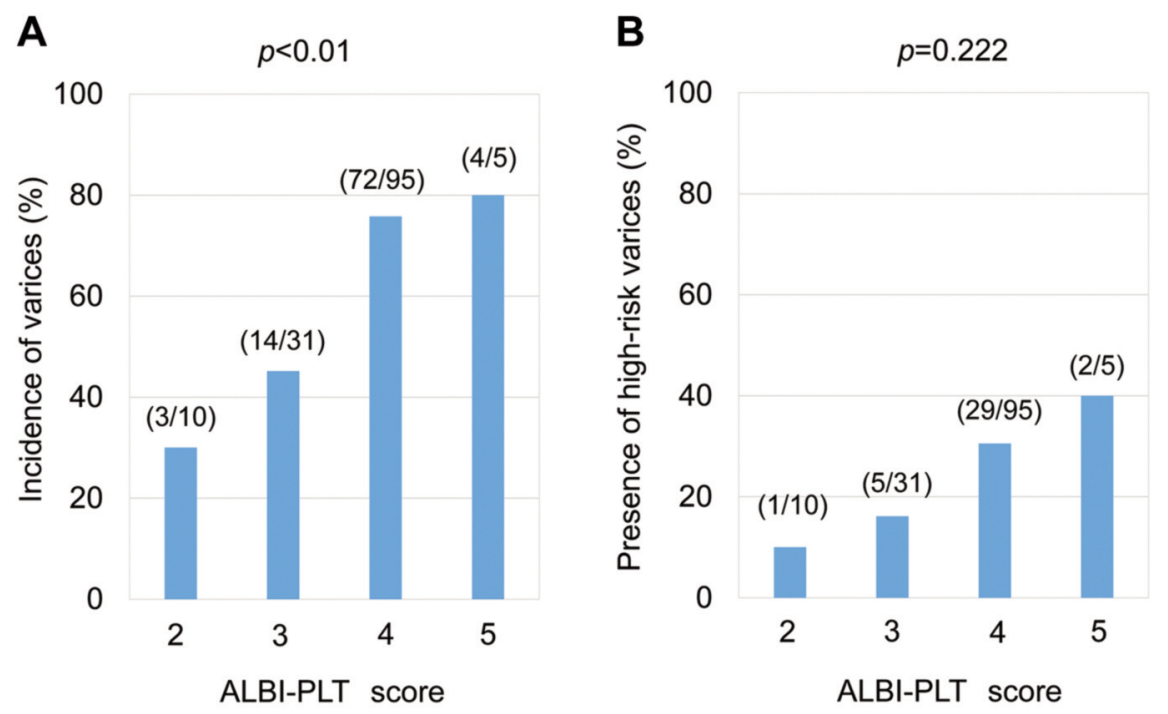

Figure 1. Association of the albumin-bilirubin (ALBI)-platelet (PLT) score with the endoscopic findings of gastroesophageal varices (GEVs). A: The incidence of GEVs increased as the score increased (overall: $p<0.01)$. B: The incidence of high-risk varices also increased as the score increased; however, the differences among the groups (score 2-5) did not reach statistical significance (overall: $p=0.222$ ).
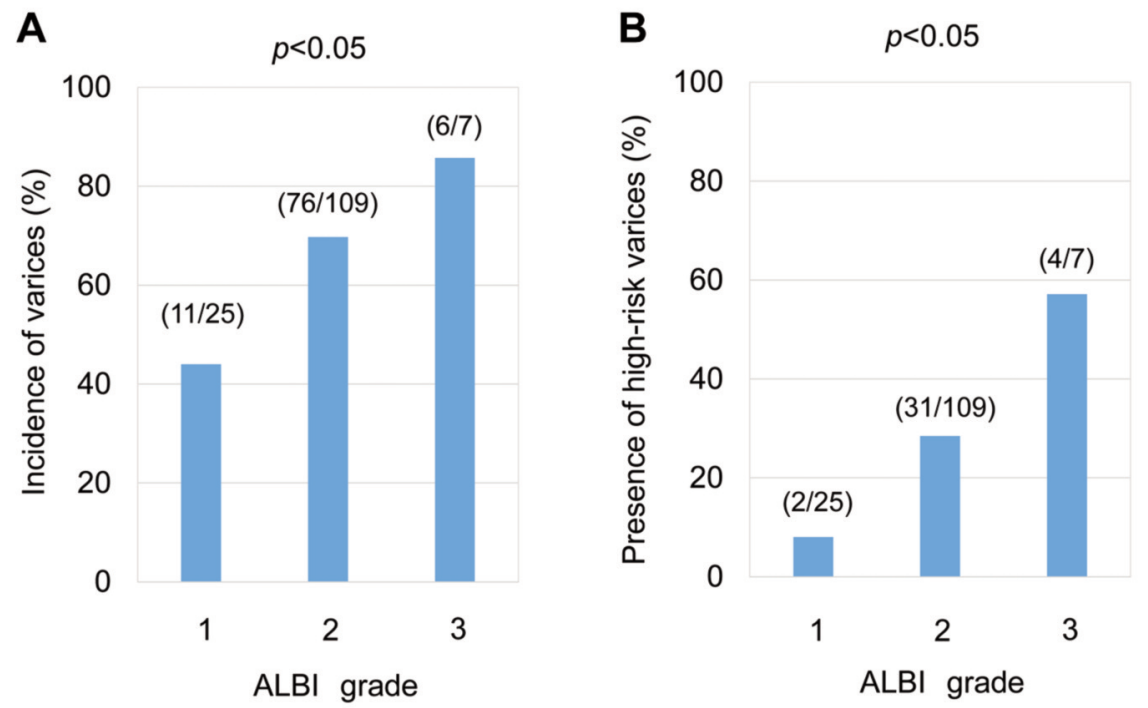

Figure 2. Association of the albumin-bilirubin (ALBI) grade with the endoscopic findings of gastroesophageal varices (GEVs). A: The incidence of GEVs among the ALBI grade groups differed statistically significantly (overall: $p<0.05$ ). B: The incidence of high-risk varices was statistically significantly increased as the grade increased (overall: $p<0.05$ ).

(overall: $p<0.01$ ) (Figure 3A) and the incidence of high-risk varices (overall: $p<0.05$ ) (Figure $3 \mathrm{~B}$ ). We further evaluated the relevance of the sub-classification of ALBI grade 2 to the endoscopic findings. The incidence of GEVs in patients with grade $2 b$ or 3 was significantly higher in comparison to those with grades 1 or $2 \mathrm{a}(p<0.0001)$ (Figure 4A). Similar results were observed regarding the incidence of high-risk GEVs $(p<0.001)$ (Figure 4B).
The incidence rates of GEVs in patients with ALBI grades 2 or 3 were significantly higher in comparison to those with ALBI grade 1 (Table II); however, when the enrolled patients were classified according to the mALBI grade, the incidence rates in patients with mALBI grade $2 \mathrm{~b}$ or grade 3 , but not those with mALBI grade $2 \mathrm{a}$, were significantly higher in comparison to that of patients with grade 1. Similar results were also observed regarding the incidence of high-risk GEVs (Table III). 

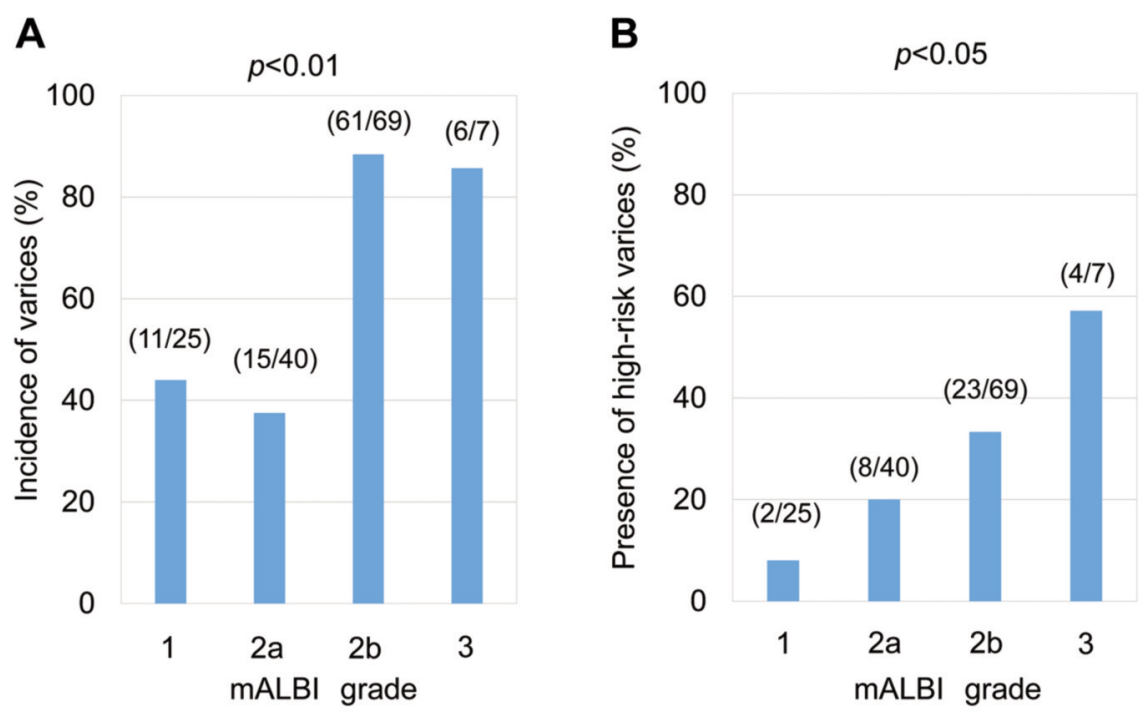

Figure 3. Association of the modified albumin-bilirubin (mALBI) grade with the endoscopic findings of gastroesophageal varices (GEVs). A: The incidence of GEVs among the mALBI grade groups was statistically significantly different (overall: $p<0.01$ ). B: Similarly, the incidence of highrisk varices among the groups differed statistically significantly (overall: $p<0.05$ ).
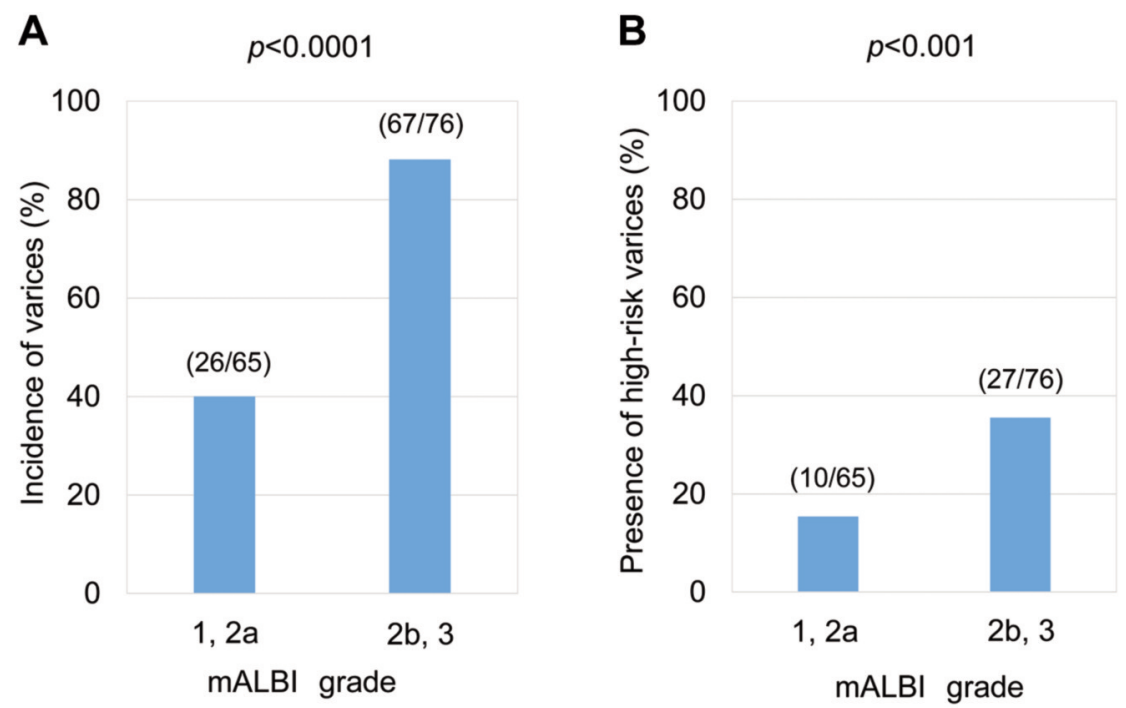

Figure 4. Comparison of the endoscopic findings of gastroesophageal varices (GEVs) between patients with albumin-bilirubin (ALBI) grade 1-2a and those with ALBI grade 2b-3. A: The incidence of GEVs in patients with grades $2 b$ and 3 was significantly higher than that in those with grades 1 and $2 a(p<0.0001)$. B: Similar results were observed regarding the incidence of high-risk GEVs $(p<0.001)$.

\section{Discussion}

Variceal bleeding is a serious complication in patients with liver cirrhosis. However, EGD is relatively burdensome, and the ability to predict GEVs using noninvasive biomarkers would be useful. The ALBI score is suggested to be a more useful evaluation than the Child-Pugh classification, especially in patients with compensated cirrhosis $(7,9)$. On the other hand, the ALBI score alone seems to have a limitation in that a large number of patients are classified into ALBI grade 2, and therefore the mALBI grade was proposed as a more detailed classification for patients with cirrhosis (9).

In 2018, a score that combined ALBI with the platelet count was proposed, and it was found that GEVs with a bleeding risk were rarely detected in patients with an ALBI-PLT score 
Table II. Association of the albumin-bilirubin (ALBI) grade and modified ALBI (mALBI) grade with the presence of gastroesophageal varices. ALBI grades and modified mALBI grades were entered as categorical variables, and odds ratios were determined.

\begin{tabular}{lccr}
\hline & Grade & Odds ratio $(95 \% \mathrm{CI})$ & $p$-Value \\
\hline ALBI grade & 1 & 1 (Reference) & \\
& 2 & $2.931(1.211-7.274)$ & $\mathbf{0 . 0 1 7 3}$ \\
mALBI grade & 3 & $7.636(1.083-155.7)$ & $\mathbf{0 . 0 4 0 5}$ \\
& 1 & 1 (Reference) & \\
& $2 \mathrm{a}$ & $0.764(0.275-2.127)$ & 0.6034 \\
& $2 \mathrm{~b}$ & $9.705(3.397-29.99)$ & $<\mathbf{0 . 0 0 0 1}$ \\
& 3 & $7.636(1.083-155.7)$ & $\mathbf{0 . 0 4 0 5}$ \\
\hline
\end{tabular}

CI: Confidence interval. Statistically significant $p$-values are shown in bold.

of 2 points (8). This suggested that the ALBI-PLT score can be used for the risk stratification of patients with ALBI grade 2 , which includes a wide range of patients. The report focused on compensated cirrhosis with liver cancer; however, consistent results were obtained in the current cohort of patients without liver cancer (Figure 1). We herein used the mALBI classification to avoid the problem that a large number of patients were classified into ALBI grade 2. To our knowledge, this is the first report to study the association of endoscopic findings with mALBI grades. The endoscopic findings of GEVs were considered to differ between patients with mALBI grade $2 \mathrm{a}$ and those with grade $2 \mathrm{~b}$ (Figure 4 ). The differences between the patients with mALBI grade 1 and those with grade $2 \mathrm{a}$ did not reach statistical significance (Tables II and III). Our findings suggest that the mALBI grade, in which grade 2 is divided into grade $2 \mathrm{a}$ and grade $2 \mathrm{~b}$, may be useful in predicting the presence of GEVs and for stratifying the bleeding risk of GEVs.

The present study was associated with several limitations. Firstly, the study population was limited to patients who underwent a liver biopsy and who had histologically proven cirrhosis. Thus, the study population was relatively small. In addition, we did not conduct liver biopsies for patients with highly advanced cirrhosis with signs of hepatic failure, such as ascites, and the study population only included a relatively small number of patients with ALBI grade 3. Secondly, all chronic liver diseases were included, and the difference due to etiology was not evaluated. Finally, in general, no single biomarker was able to completely predict the endoscopic findings of GEVs (15-17), and a combination of mALBI with other variable/parameter(s) should be considered.

In summary, we evaluated the association of the mALBI score with the endoscopic findings of GEVs. The endoscopic findings of GEVs were considered to differ between patients with mALBI grade $2 \mathrm{a}$ and those with grade $2 \mathrm{~b}$. The incidence of GEVs predicted by the mALBI score, with the
Table III. Association of the albumin-bilirubin (ALBI) grade and modified ALBI (mALBI) grade with the presence of high-risk gastroesophageal varices. ALBI grades and $m A L B I$ grades were entered as categorical variables, and odds ratios were determined.

\begin{tabular}{lccc}
\hline & Grade & Odds ratio $(95 \% \mathrm{CI})$ & $p$-Value \\
\hline ALBI grade & 1 & 1 (Reference) & \\
& 2 & $4.571(1.247-29.54)$ & $\mathbf{0 . 0 1 9 0}$ \\
mALBI grade & 3 & $15.33(2.127-156.5)$ & $\mathbf{0 . 0 0 6 6}$ \\
& 1 & 1 (Reference) & \\
& $2 \mathrm{a}$ & $2.875(0.646-20.23)$ & 0.1748 \\
& $2 \mathrm{~b}$ & $5.750(1.516-37.78)$ & $\mathbf{0 . 0 0 7 7}$ \\
& 3 & $15.33(2.127-156.5)$ & $\mathbf{0 . 0 0 6 6}$ \\
\hline
\end{tabular}

CI: Confidence interval. Statistically significant $p$-values are shown in bold.

sub-classifications of grade $2 \mathrm{a}$ and $2 \mathrm{~b}$, may differ from that predicted by ALBI grade 2 .

\section{Conflicts of Interest}

The Authors declare no conflicts of interest in association with the present study.

\section{Authors' Contributions}

YM collected and analyzed the data; HE designed the study, analyzed the data and wrote the article. NH, TN, and YI collected the data; SN and HI collected the data and supervised the study. All Authors reviewed and edited the article, and approved the final version of the article.

\section{Acknowledgements}

This work was partially funded by a grant from the Hyogo Innovative Challenge, Hyogo College of Medicine, Japan.

The Authors are grateful to Inui S, Higuchi Y, Kanazawa N, Matsushita Y, Fujii S, Kido H and Minemoto K (Hyogo College of Medicine) for their technical and secretarial assistance.

\section{References}

1 Garcia-Tsao G, Abraldes JG, Berzigotti A and Bosch J: Portal hypertensive bleeding in cirrhosis: Risk stratification, diagnosis, and management: 2016 Practice guidance by the American Association for the Study of Liver Diseases. Hepatology 65(1): 310-335, 2017. PMID: 27786365. DOI: 10.1002/hep.28906

2 European Association for the Study of the Liver: EASL Clinical Practice Guidelines for the management of patients with decompensated cirrhosis. J Hepatol 69(2): 406-460, 2018. PMID: 29653741. DOI: 10.1016/j.jhep.2018.03.024

3 Fukui H, Saito H, Ueno Y, Uto H, Obara K, Sakaida I, Shibuya A, Seike M, Nagoshi S, Segawa M, Tsubouchi H, Moriwaki H, Kato A, Hashimoto E, Michitaka K, Murawaki T, Sugano K, Watanabe $\mathrm{M}$ and Shimosegawa T: Evidence-based clinical 
practice guidelines for liver cirrhosis 2015. J Gastroenterol 51(7): 629-650, 2016. PMID: 27246107. DOI: 10.1007/s00535016-1216-y

4 Lee HA, Kim SU, Seo YS, Lee YS, Kang SH, Jung YK, Kim MY, Kim JH, Kim SG, Suk KT, Jung SW, Jang JY, An H, Yim $\mathrm{HJ}$ and $\mathrm{Um} \mathrm{SH}$ : Prediction of the varices needing treatment with non-invasive tests in patients with compensated advanced chronic liver disease. Liver Int 39(6): 1071-1079, 2019. PMID: 30589490. DOI: 10.1111/liv.14036

5 Kim NJ, Su F and Biggins SW: CON: Noninvasive modalities are preferred to screen for the diagnosis of esophageal and gastric varices when the diagnosis of cirrhosis is made. Clin Liver Dis 16(2): 48-52, 2020. PMID: 32922749. DOI: 10.1002/ cld .952

6 de Franchis R; Baveno VI Faculty: Expanding consensus in portal hypertension: Report of the Baveno VI Consensus Workshop: Stratifying risk and individualizing care for portal hypertension. J Hepatol 63(3): 743-752, 2015. PMID: 26047908. DOI: $10.1016 /$ j.jhep.2015.05.022

7 Johnson PJ, Berhane S, Kagebayashi C, Satomura S, Teng M, Reeves HL, O’Beirne J, Fox R, Skowronska A, Palmer D, Yeo W, Mo F, Lai P, Iñarrairaegui M, Chan SL, Sangro B, Miksad R, Tada T, Kumada T and Toyoda H: Assessment of liver function in patients with hepatocellular carcinoma: A new evidence-based approach - the ALBI grade. J Clin Oncol 33(6): 550-558, 2015. PMID: 25512453. DOI: 10.1200/JCO.2014.57.9151

8 Chen PH, Hsieh WY, Su CW, Hou MC, Wang YP, Hsin IF, Yang TC, Liao WC, Lin HC, Lee FY and Wu JC: Combination of albumin-bilirubin grade and platelets to predict a compensated patient with hepatocellular carcinoma who does not require endoscopic screening for esophageal varices. Gastrointest Endosc 88(2): 230-239.e2, 2018. PMID: 29317268. DOI: 10.1016/j.gie.2017.12.023

9 Hiraoka A, Kumada T, Tsuji K, Takaguchi K, Itobayashi E, Kariyama K, Ochi H, Tajiri K, Hirooka M, Shimada N, Ishikawa T, Tachi Y, Tada T, Toyoda H, Nouso K, Joko K, Hiasa Y, Michitaka $\mathrm{K}$ and Kudo M: Validation of modified ALBI grade for more detailed assessment of hepatic function in hepatocellular carcinoma patients: A multicenter analysis. Liver Cancer 8(2): 121-129, 2019. PMID: 31019902. DOI: $10.1159 / 000488778$

10 Enomoto H, Ueno Y, Hiasa Y, Nishikawa H, Hige S, Takikawa Y, Taniai M, Ishikawa T, Yasui K, Takaki A, Takaguchi K, Ido A, Kurosaki M, Kanto T, Nishiguchi S; Japan Etiology of Liver Cirrhosis Study Group in the 54th Annual Meeting of JSH: Transition in the etiology of liver cirrhosis in Japan: A nationwide survey. J Gastroenterol 55(3): 353-362, 2020. PMID: 31768801. DOI: 10.1007/s00535-019-01645-y
11 Shimono Y, Enomoto H, Kishino K, Moriwaki EI, Nishikawa H, Nishimura T, Iwata Y, Iijima $\mathrm{H}$ and Nishiguchi S: Arm skeletal muscle mass is associated with the prognosis of patients with cirrhosis. In Vivo 34(3): 1165-1171, 2020. PMID: 32354906. DOI: 10.21873 /invivo.11889

12 Moriwaki EI, Enomoto H, Saito M, Hara N, Nishikawa H, Nishimura $\mathrm{T}$, Iwata $\mathrm{Y}$, Iijima $\mathrm{H}$ and Nishiguchi $\mathrm{S}$ : The anthropometric assessment with the bioimpedance method is associated with the prognosis of cirrhotic patients. In Vivo 34(2): 687-693, 2020. PMID: 32111771. DOI: 10.21873/invivo.11825

13 Yuri Y, Nishikawa H, Enomoto H, Yoh K, Iwata Y, Sakai Y, Kishino K, Ikeda N, Takashima T, Aizawa N, Takata R, Hasegawa K, Ishii N, Nishimura T, Iijima $\mathrm{H}$ and Nishiguchi S: Impact of sustained virological response for gastroesophageal varices in hepatitis-C-virus-related liver cirrhosis. J Clin Med 9(1): 95, 2019. PMID: 31905953. DOI: 10.3390/jcm9010095

14 Nishikawa H, Yoh K, Enomoto H, Iwata Y, Sakai Y, Kishino K, Shimono Y, Ikeda N, Takashima T, Aizawa N, Takata R, Hasegawa K, Koriyama T, Yuri Y, Nishimura T, Nishiguchi S and Iijima H: Sarcopenia and frailty in chronic liver damage: Common and different points. In Vivo 34(5): 2549-2559, 2020. PMID: 32871784. DOI: 10.21873/invivo.12072

15 Lesmana CRA, Raharjo $M$ and Gani RA: Managing liver cirrhotic complications: Overview of esophageal and gastric varices. Clin Mol Hepatol 26(4): 444-460, 2020. PMID: 33053928. DOI: $10.3350 / \mathrm{cmh} .2020 .0022$

16 Pateu E, Oberti $\mathrm{F}$ and Calès $\mathrm{P}$ : The noninvasive diagnosis of esophageal varices and its application in clinical practice. Clin Res Hepatol Gastroenterol 42(1): 6-16, 2018. PMID: 28870440. DOI: 10.1016/j.clinre.2017.07.006

17 Calvaruso V, Cacciola I, Licata A, Madonia S, Benigno R, Petta S, Bronte F, Conte E, Malizia G, Bertino G, Distefano M, Montineri A, Digiacomo A, Alaimo G, Cacopardo B, Davì A, Guarneri L, Scalisi I, Colletti P, Cartabellotta F, Portelli V, Prestileo T, Averna A, Iacobello C, Mondello L, Scifo G, Russello M, Squadrito G, Raimondo $\mathrm{G}$, Cammà C, Craxì $\mathrm{A}$, Di Marco V and RESIST-HCV (Rete Sicilia Selezione Terapia$\mathrm{HCV}$ ): Is transient elastography needed for noninvasive assessment of high-risk varices? The REAL experience. Am J Gastroenterol 114(8): 1275-1282, 2019. PMID: 31135449. DOI: 10.14309/ajg.0000000000000266

Received December 4, 2020

Revised December 20, 2020

Accepted December 21, 2020 